\title{
SOME NONLOCAL RESULTS FOR WEAKLY NONLINEAR DYNAMICAL SYSTEMS*
}

\author{
BY \\ P. R. SETHNA AND T. J. MORAN \\ University of Minnesota
}

I. Introduction. There is a large amount of mathematical literature on weakly nonlinear dynamical systems, i.e., systems of the type:

$$
\dot{y}=A y+\epsilon g(t, y)
$$

where $y$ is a real $n$ vector, $\epsilon$ a small real parameter, $A$ an $n \times n$ real matrix, and $g$ an $n$ vector function either periodic or almost periodic in $t$. If the matrix $A$ has pure imaginary characteristic roots with simple elementary divisors, it is often more convenient to study the related systems, obtained by the method of variation of constants from (1.1), in the following form:

$$
\dot{x}=\epsilon f(t, x)
$$

where $x$ is an $n$ vector, $\epsilon$ the same small parameter as in (1.1) and $f$ an $n$ vector function periodic or almost periodic in $t$.

The results in the literature, valid for all time, for either systems (1.1) or (1.2), are concerned with the existence and stability of periodic or almost periodic solutions and usually a technique for obtaining approximations to these solutions is also given. See, for example, the books by Hale [1], Coddington and Levinson [2], and Bogoliuboff and Mitropolskiy [3].

Bogoliuboff and Mitropolskiy [4] also discuss solutions other than periodic or almost periodic solutions, for systems of the type (1.2), by comparing the solution of (1.2) with the solution of the associated "averaged equation", but their results are valid only for a finite interval of time.

Our study is concerned with the solutions of (1.2), valid for all time, but which are not restricted to only periodic or almost periodic solutions. Our main result gives sufficient conditions for the solutions of (1.2) to approach stable almost periodic solutions of (1.2) as time goes to infinity. As in [4], we also compare the solutions of (1.2) with the solutions of the associated "averaged equations" but in our case asymptotically valid estimates for solutions of (1.2) are given for all time.

On the basis of this main result, it is possible to give, with the aid of a theorem of LaSalle [5], results regarding the domains of attraction of almost periodic solutions of (1.2). These results regarding the domains of attraction are generalizations of the results for periodic systems given by Loud and Sethna in [6]. In [6] explicit estimates are given for the domains of attraction for second order systems. In the present study we give explicit estimates for the domains of attraction of almost periodic solutions of dynamical systems of order greater then two but in the special case of systems

${ }^{*}$ Received March 29, 1967. This research was supported by the United States Air Force Office of Scientific Research under Grant AF-AFOSR-704-66. 
with polynomial nonlinearities and when the system is performing "monofrequent oscillations" [7].

II. A nonlocal result. Consider the real system of differential equations:

$$
\dot{x}=\epsilon f(t, x, \epsilon)
$$

where $x$ is an $n$ vector, $0 \leq \epsilon \leq \epsilon_{0}$, and $f$ is a function from:

$B\left(K, \epsilon_{0}\right)=\left\{(t, x, \epsilon): x\right.$ an $n$ vector, $t$ and $\epsilon$ scalars, $0 \leq \epsilon \leq \epsilon_{0},-\infty<t<\infty$, $|x| \leq K\}$ into $E^{n}$. It will be assumed throughout that:

(i) $f$ is bounded and has bounded first partial derivatives with respect to $x$,

(ii) $f$ is almost periodic in $t$ uniformly in $x$ for each fixed $\epsilon$.

Consider also the averaged system of equations

$$
\dot{\xi}=\epsilon f_{0}(\xi)
$$

where

$$
f_{0}(\xi)=\lim _{T \rightarrow \infty} T^{-1} \int_{0}^{T} f(t, \xi, 0) d t .
$$

Bogoliuboff and Mitropolskiy [4] in their discussion of the method of averaging give results relating the solutions of (2.1) and the corresponding solutions of the averaged equations (2.2). Since our main result is based on two theorems of Bogoliuboff and Mitropolskiy, we will give statements of these theorems for reference.

One of these theorems gives a result valid for a finite time interval. The statement of the theorem is as follows:

Theorem A. If system (2.1) defined on $B\left(K, \epsilon_{0}\right)$ satisfies conditions (i) and (ii), then given $\rho>0, \eta>0$, however small, and $L>0$, however large, there exists an $\epsilon_{1}, 0<\epsilon_{1}<\epsilon_{0}$ such that if $\xi(t)$ is a solution of (2.2) defined for $0 \leq t<\infty$, and $\xi(t)$ and its $\rho$ neighborhood remain in the set $B(K)=\{x: x$ an $n$ vector, $|x| \leq K\}$ for all $t \geq 0$, then for all $\epsilon, 0<\epsilon<\epsilon_{1}$,

$$
|x(t)-\xi(t)|<\eta
$$

for $0 \leq t<L / \epsilon$ where $x(t)$ is a solution of (2.1) with $x(0)=\xi(0) \varepsilon B(K)$.

The statement as given above is more general than the one given in [4] in that here $f$ depends explicitly on $\epsilon$ and is more restricted in that conditions (i) and (ii) are more restrictive than those in [4].

The other theorem in reference [4] that is of interest to us, and which is valid for all time, is concerned with existence and stability of almost periodic solutions of (2.1). A statement of the theorem, somewhat modified for our purposes, is as follows:

Theorem B. Let system (2.1) defined on the set $B\left(K, \epsilon_{0}\right)$ satisfy conditions (i) and (ii). Let $\xi_{0}$ be a constant solution of (2.2) which together with its $\delta$ neighborhood is in the interior of $B(K)$.

If the variational equation of (2.2) with respect to $\xi_{0}$, that is

$$
\frac{d z}{d t}=\epsilon \frac{\partial f_{0}\left(\xi_{0}\right)}{\partial \xi} z
$$

has characteristic roots with negative real parts, then there exist positive constants $\epsilon_{2}$ and 
$\sigma, 0<\epsilon_{2} \leq \epsilon_{0}, 0<\sigma<\delta$, such that for each $\epsilon, 0<\epsilon \leq \epsilon_{2}$, there is a unique almost periodic solution $x^{*}(t, \epsilon)$ of $(2.1)$, continuous in $\epsilon$, which satisfies:

(1) $\left|x^{*}(t, \epsilon)-\xi_{0}\right|<\sigma$ for $-\infty<t<\infty$,

(2) $\lim _{\epsilon \rightarrow 0}\left|x^{*}(t, \epsilon)-\xi_{0}\right|=0$ uniformly in $t$.

(3) If $x(t)$ is any solution of (2.1) which at some time $t_{0}$ satisfies

$$
\left|x\left(t_{0}\right)-\xi_{0}\right|<\sigma
$$

then we can find positive constants $C$ and $\gamma$ so that

$$
\left|x(t)-x^{*}(t, \epsilon)\right| \leq C \exp \left[-\epsilon \gamma\left(t-t_{0}\right)\right], \quad t \geq t_{0} .
$$

REMARK. In the course of the proof it is seen that the constant $\sigma$ can in fact be chosen arbitrarily small and the constant $\epsilon_{2}$ is then determined by this choice.

Our main result states sufficient conditions under which the solutions of the original system (2.1) and the solutions of the averaged system (2.2), starting initially at the same point, remain as close to each other as desired, at each $t$, for all $t$, for $\epsilon$ sufficiently small. These conditions are given in the following:

Theorem 1. Let system (2.1) be defined on the set $B\left(K, \epsilon_{0}\right)$ and satisfy conditions (i) and (ii). Let $\xi_{0}$ be a constant solution of (2.2) which, with its $\delta$ neighborhood, is in the set $B(K)=\{x: x$ an $n$ vector, $|x| \leq K\}$, and let the eigenvalues of (2.3) have negative real parts.

Given any scalar $\rho>0$, however small, let $\xi(t)$ be a solution of $(2.2)$, with $\xi(0) \varepsilon B(K)$, which, together with its $\rho$ neighborhood, remains in $B(K)$ for all $t \geq 0$ and furthermore $\lim _{t \rightarrow \infty} \xi(t)=\xi_{0}$.

Under these conditions we can conclude that given any $\eta>0$ there exists an $\epsilon^{*}, 0<\epsilon^{*} \leq \epsilon_{0}$ such that for all $\epsilon, 0<\epsilon \leq \epsilon^{*}$, the solution $x(t, \epsilon)$ of $(2.1)$ with $x(0, \epsilon)=\xi(0)$ satisfies $|x(t, \epsilon)-\xi(t)|<\eta$ for all $t \geq 0$.

Furthermore, there exists an $n$ vector function $x^{*}(t, \epsilon)$ continuous in $t$ and $\epsilon$, almost periodic in $t$, which is a solution of (2.1) and such that $\lim _{\epsilon \rightarrow 0}\left|x^{*}(t, \epsilon)-\xi_{0}\right|=0$ and $\lim _{t \rightarrow \infty}\left|x(t, \epsilon)-x^{*}(t, \epsilon)\right|=0$.

Proof. From Theorem B there exists a $\sigma>0$, which can be chosen as small as we wish, and an $\epsilon_{2}, 0<\epsilon_{2}<\epsilon_{0}$, such that for each $\epsilon, 0<\epsilon \leq \epsilon_{2}$, there exists an $n$ vector function $x^{*}(t, \epsilon)$ almost periodic in $t$ which is a solution of (2.1) with the properties (1), (2) and (3) as given in Theorem B.

Let $x(t, \epsilon)$ and $\xi(t)$ be solutions of (2.1) and (2.2) respectively such that $x(0, \epsilon)=$ $\xi(0)=x_{0} \varepsilon B(K)$. By Theorem A, choose $\epsilon_{1}$ such that, for $0<\epsilon<\epsilon_{1}$,

$$
|x(t)-\xi(t)| \leq \sigma / 2, \quad 0 \leq t<L / \epsilon,
$$

and we can always choose $\epsilon_{1}, 0<\epsilon_{1} \leq \epsilon_{2}$. Since $\xi(t) \rightarrow \xi_{0}$, as $t \rightarrow \infty$, we can always choose $L>0$ such that the solution of the averaged equation

$$
d \xi / d \tau=f_{0}(\xi)
$$

has the property that for any given $\theta>0$

$$
\left|\xi(\tau)-\xi_{0}\right| \leqq \theta / 2 \quad \text { for } \quad \tau \geqq L / 2 .
$$

Now let $\tau=\epsilon t$ and $\theta=\sigma$ of Theorem B; then we have 


$$
\left|\xi(t)-\xi_{0}\right| \leqq \sigma / 2
$$

for $t \geq L / 2 \epsilon$, for $0<\epsilon<\epsilon_{1}$. For any $t$ we have:

$$
\left|x(t)-\xi_{0}\right| \leq|x(t)-\xi(t)|+\left|\xi(t)-\xi_{0}\right| .
$$

Now, if $L / 2 \epsilon \leq t<L / \epsilon$, then from (2.4) and (2.5) we have

$$
\left|x(t)-\xi_{0}\right|<\sigma .
$$

By properties (1) and (3) of Theorem B we can show that if, for some $t=t_{0}$,

$$
\left|x\left(t_{0}\right)-\xi_{0}\right|<\sigma
$$

then

$$
\left|x(t)-x^{*}(t, \epsilon)\right| \leq 2 \sigma, \quad t \geq t_{0} .
$$

For all $t$ we have:

$$
\left|x(t)-\xi_{0}\right| \leq\left|x(t)-x^{*}(t, \epsilon)\right|+\left|x^{*}(t, \epsilon)-\xi_{0}\right| .
$$

Using property (1) of Theorem B and (2.6) we have from (2.7) for $t \geq t_{0}$,

$$
\left|x(t)-\xi_{0}\right| \leq 3 \sigma \text {. }
$$

Now take $t_{0}=L / 2 \epsilon$; then

$$
\left|x(t)-\xi_{0}\right| \leq 3 \sigma, \quad L / 2 \epsilon \leq t<\infty .
$$

Now consider

$$
|x(t)-\xi(t)| \leq\left|x(t)-\xi_{0}\right|+\left|\xi(t)-\xi_{0}\right|
$$

then from (2.8) and (2.5), for $L / 2 \epsilon<t<\infty$, we have

$$
|x(t)-\xi(t)| \leq 3 \sigma+\sigma / 2 .
$$

Thus

$$
|x(t)-\xi(t)| \leq 7 / 2 \sigma, \quad L / 2 \epsilon<t<\infty,
$$

and from (2.4) we have

$$
|x(t)-\xi(t)| \leq \sigma / 2, \quad 0 \leq t<L / \epsilon .
$$

Thus if we choose $\sigma=2 \eta / 7$ and $\epsilon_{1}=\epsilon^{*}$ we have the desired result.

Example. As an application of the theorem consider the van der Pol equation:

$$
\ddot{x}+\epsilon\left(x^{2}-1\right) \dot{x}+x=0 .
$$

Let

$$
\begin{aligned}
& x(t)=A(t) \cos \Psi(t), \\
& \dot{x}(t)=-A(t) \sin \Psi(t), \quad A \neq 0 .
\end{aligned}
$$

Then

$$
\begin{aligned}
& \dot{A}=-\epsilon\left(A^{2} \cos ^{2} \Psi-1\right) A \sin ^{2} \Psi, \\
& \dot{\Psi}=1-\epsilon\left(A^{2} \cos ^{2} \Psi-1\right) \sin \Psi \cos \Psi .
\end{aligned}
$$

Now consider $\Psi$ as the new independent variable and observe that for $\epsilon$ sufficiently small we can write system (2.10) in the form 


$$
\frac{d A}{d \Psi}=-\epsilon \frac{\left(A^{2} \cos ^{2} \Psi-1\right) A \sin ^{2} \Psi}{1-\epsilon\left(A^{2} \cos ^{2} \Psi-1\right) \sin \Psi \cos \Psi}
$$

which corresponds to Eq. (2.1) in Theorem 1 and satisfies all the conditions on the function $f$ of the theorem. Let $a$ be the averaged variable corresponding to $A$ and we obtain the averaged equation:

$$
\frac{d a}{d \Psi}=-(\epsilon / 8) a\left(a^{2}-4\right)
$$

which can be solved to yield

$$
a^{2}=4\left\{1-\frac{a_{0}^{2}-4}{a_{0}^{2}} \exp \left[-\epsilon\left(\Psi-\Psi_{0}\right)\right]\right\}^{-1}
$$

where $a_{0}=a\left(\Psi_{0}\right)$.

We observe that (2.11) has an asymptotically stable constant solution $a=2$ and from (2.12) we have that all solutions with initial condition other than $a_{0}=0$ approach this solution monotonically in $\Psi$. Furthermore, from the second equation in (2.10) we can conclude that for $\epsilon$ sufficiently small, $\Psi(t)$ is a monotone increasing function of $t$ and $\Psi(t) \rightarrow \infty$ as $t \rightarrow \infty$, so that $\Psi(t)$ behaves essentially like time.

Now from Theorem 1 we conclude that $A(\Psi, \epsilon)$ remains arbitrarily close to $a(\Psi)$ for all $\Psi$ and approaches an almost periodic solution arbitrarily close to $a=2$ as $\Psi \rightarrow \infty$. While this result does not give an estimate of $x$ as a function of $t$, it does give an estimate of the trajectories in the $(x, \dot{x})$ phase space. This we see from a polar plot of $a(\Psi)$ with $\Psi$ the angle variable. This plot is an approximation to the trajectories in the $(x, \dot{x})$ phase space, and in this space all solutions other than $x=\dot{x}=0$ approach the limit cycle $x^{2}+\dot{x}^{2}=2$ like logarithmic spirals.

In most applications, it is not possible to obtain an explicit solution $\xi(t)$ of (2.2) for arbitrary initial conditions. Useful information, however, can be obtained if one can compare the domain of attraction of an asymptotically stable constant solution $\xi_{0}$ of (2.2) with the domain of attraction of the corresponding almost periodic solution $x^{*}(t, \epsilon)$ of $(2.1)$. Such a result can be obtained from the following corollary to the basic theorem.

Corollary. Let $D_{0} \subset B(K)$ be a domain of attraction of $\xi_{0}$. Then if $D$ is any compact subset of $D_{0}$ such that for all $\xi(t)$ with $\xi(0) \varepsilon D, \xi(t)$ does not reach the boundary of $D_{0}$, then there exists an $\epsilon^{*}>0$ such that for all $\epsilon, 0<\epsilon<\epsilon^{*}, D$ is contained in the domain of attraction of $x^{*}(t, \epsilon)$.

Proof. Let $S=\left\{\xi(t): \xi(t)\right.$ is a solution of $\xi=\epsilon f_{0}(\xi)$ with $\left.\xi(0) \varepsilon D\right\}$.

Let

$$
\rho=\inf _{\xi(t) \varepsilon S} \min _{t \geq 0}\left|\xi(t)-\partial D_{0}\right| .
$$

Since $D$ is compact, the above infimum is attained for some $\xi(t) \varepsilon S$. Thus $\rho>0$. By the theorem, then, there exists an $\epsilon^{*}$ such that for all $\epsilon, 0<\epsilon<\epsilon^{*}, x(t, \epsilon) \rightarrow x^{*}(t, \epsilon)$ as $t \rightarrow \infty$ for all $x(0, \epsilon) \varepsilon D$. This completes the proof.

The problem thus reduces to that of finding the domain of attraction $D_{0}$ and its compact subset $D$ for each asymptotically stable constant solution $\xi_{0}$ of (2.2). For this purpose we use a result of LaSalle [6] which will be given here for reference. 
Theorem (LaSalle). Let $V(x)$ be a scalar function with continuous first partial derivatives. Let $\Omega_{l}$ denote the region where $V(x)<l$. Assume that $\Omega_{l}$ is bounded and that within $\Omega_{l}, V(x)>0$ for all $x \neq 0$. Furthermore, let $\dot{V}(x)<0$ for all $x \neq 0$, in $\Omega_{l}$. Then the origin is asymptotically stable, and every solution in $\Omega_{l}$ tends to the origin as $t \rightarrow \infty$.

If we take for the set $D_{0}$ in the Corollary the set $\Omega_{l}$ in IaSalle's theorem, and take for the set $D$ the set

$$
\bar{\Omega}_{l-\delta}=\{x: V(x) \leq l-\delta\}
$$

for $0<\delta<l$, then we can establish the desired relationship between the domains of attraction of $\xi_{0}$ and $x^{*}(t, \epsilon)$.

III. Domains of attraction for monofrequent systems. In [6] a method was given to obtain explicit estimates of domains of attraction of periodic solutions of quite general weakly nonlinear second order systems. In this study we will give a method for obtaining explicit estimates of domains of attraction of almost periodic solutions of a restricted class of $n$th order weakly nonlinear systems.

We will study motions in finite neighborhoods of stable static equilibrium configurations of the system. We will assume the system to have polynomial nonlinearities, to be periodic in time, and to have nonconservative forces that are truly dissipative. In addition the system will be assumed to have no "internal resonance" [8]. (A sufficient but much stronger restriction is that the linear natural frequencies are linearly independent over the integers.)

Many dynamical systems of physical importance satisfy these conditions. Similar systems have been described as "monofrequent systems" by Mitropolskiy [3], [9], and he has studied them in detail for their local behavior.

Systems of this type have relatively simple and interesting properties. The motion for any given initial condition, as $t \rightarrow \infty$, tends to become almost periodic but with a dominant term that is periodic, with a period that is rationally related to the system period, and the general behavior of systems of this type, as $t \rightarrow \infty$, is similar to the behavior of a related second order system. The averaged equations for such systems also have certain interesting properties. The critical points for the averaged system lie in only one plane of the phase space which we will call the principal plane. All solutions of the averaged equations starting in this plane remain in this plane for all time.

We will, in the following analysis, demonstrate the above properties. Furthermore, we will obtain domains of attraction of asymptotically stable constant solutions of the averaged equations in their $n$ dimensional phase space. These domains of attraction will have the property that their projections on the principal plane are domains of attraction in the plane.

With the aid of this information regarding the domains of attraction of the constant solution of the averaged equations, we can get explicit estimates of the domains of attraction of the almost periodic solutions associated with these constant solutions by using the Corollary to Theorem 1.

Specifically, consider a $2 N$ order dynamical system described by the Lagrange's equations of motion:

$$
\frac{d}{d t}\left(\mathscr{L}_{. \dot{\alpha}_{i}}\right)-\mathscr{L}_{. a_{i}}=-\epsilon F_{i}(q, \dot{q}), \quad j=1,2, \cdots N
$$

where $q_{i}$ and $\dot{q}_{i}$ are components of $N$ vectors, $\epsilon$ is a scalar $0<\epsilon \ll 1$. A dot over a symbol 
represents differentiation with respect to $t$ and a comma followed by a variable means partial differentiation with respect to that variable. The functions

$$
F_{j}(q, \dot{q}) \equiv F_{j}\left(q_{1}, q_{2}, \cdots q_{N}, \dot{q}_{1}, \dot{q}_{2}, \cdots \dot{q}_{N}\right), \quad j=1,2, \cdots N
$$

represent the nonlinear dissipative forces. Each $F_{i}$ will be assumed to be a polynomial in $q$ and $\dot{q}$. It will be further assumed that these dissipative forces be truly dissipative, that is, the average work done:

$$
\lim _{T \rightarrow \infty} T^{-1} \int_{0}^{T} F_{i}(q(t), \dot{q}(t)) \dot{q}_{i}(t) d t, \quad j=1,2,3, \cdots N,
$$

is greater than zero for $q_{i}(t) \not \equiv 0, \dot{q}_{i}(t) \not \equiv 0$. In (3.1) the function $\&$ has the special form:

$$
\mathcal{L}(q, \dot{q}, t)=\sum_{i=1}^{N} \frac{1}{2}\left(\dot{q}_{i}^{2}+\omega_{i}^{2} q_{i}^{2}\right)+\epsilon \mathcal{L}^{1}(q, \dot{q}, t)
$$

where $\mathscr{L}^{1}$ is a polynomial in $q$ and $\dot{q}$ and is periodic in $t$ of period $2 \pi / \nu$, with $\nu$ a scalar. The scalars $\omega_{i}>0, j=1,2, \cdots N$, are the linear natural frequencies of the system and it is sufficient to assume that they are linearly independent over the integers. This condition is by no means necessary. For example in [8], for fourth order systems with cubic nonlinear terms, a necessary and sufficient condition for the system to have monofrequent motions is that the ratio of the two natural frequencies be not equal to, or be in $\epsilon$ neighborhoods of, the numbers 1,3 or $1 / 3$. With regard to the frequencies $\omega_{i}, j=1,2, \cdots N$ it will be further assumed that there is one frequency such that $r \omega_{1}+s \nu=0$ for some integers $r$ and $s$. The frequency $\omega_{1}$ is not necessarily the lowest of the natural frequencies.

In problems concerning domains of attraction, it is desirable to use a Hamiltonian formulation of the problem. We will therefore introduce momenta $p_{i}$ conjugate to the coordinates $q_{i}, j=1,2, \cdots, N$ as follows:

$$
p_{i}=\mathscr{L}_{, \dot{e}_{i}}=\dot{q}_{i}+\epsilon \mathcal{L}_{, \dot{q}_{i}}^{1}
$$

and the Hamiltonian is then:

$$
H=\sum_{i=1}^{N} p_{i} \dot{q}_{i}-\mathcal{L}(q, \dot{q}, t) .
$$

Substituting for $\dot{q}$ from (3.4) in (3.5) we have

$$
H(p, q, t)=\frac{1}{2} \sum_{i=1}^{N}\left(p_{i}^{2}+\omega_{i}^{2} q_{i}^{2}\right)+\epsilon H^{1}(q, p, t)+O\left(\epsilon^{2}\right) .
$$

We thus have the canonical equations

$$
\begin{aligned}
& \dot{q}_{i}=p_{i}+\epsilon H_{p_{i}}^{1}+O\left(\epsilon^{2}\right), \\
& \dot{p}_{i}=-\omega_{i}^{2} q_{i}-\epsilon\left[H_{,{ }_{i} i}^{1}+\epsilon f_{i}(q, p)\right]+O\left(\epsilon^{2}\right) .
\end{aligned}
$$

For $\epsilon=0$, (3.6) has the solutions:

$$
\begin{aligned}
& q_{i}=\omega_{i}^{-1 / 2}\left(Q_{i} \cos \omega_{i} t+P_{i} \sin \omega_{i} t\right), \\
& p_{i}=\omega_{i}^{1 / 2}\left(-Q_{i} \sin \omega_{j} t+P_{i} \cos \omega_{i} t\right)
\end{aligned}
$$

or alternatively 


$$
\begin{aligned}
q_{i} & =\omega_{i}^{-1}\left(2 R_{i}\right)^{1 / 2} \cos \left[\omega_{i}\left(t-\Phi_{j}\right)\right], \\
p_{i} & =-\left(2 R_{j}\right)^{1 / 2} \sin \left[\omega_{i}\left(t-\Phi_{i}\right)\right], \quad R_{i} \neq 0
\end{aligned}
$$

where $(Q, P)$ and $(R, \Phi)$ are constants.

We can now regard (3.7) and (3.8) as transformations $(p, q) \leftrightarrow(P, Q)$ or $(p, q) \leftrightarrow(R, \Phi)$ respectively. Transformation (3.7) is similar to the well-known van der Pol transformation and (3.8) is similar to the Kryloff and Bogoliuboff transformation with the difference that (3.7) and (3.8) are canonical. Substituting (3.7) into (3.6) we have

$$
\begin{aligned}
& \dot{Q}_{i}=\epsilon\left[K_{, P_{i}}+\left(\omega_{i}^{-1 / 2} \sin \omega_{i} t\right) f_{j}(P, Q, t)\right]+O\left(\epsilon^{2}\right), \\
& \dot{P}_{i}=-\epsilon\left[K_{Q_{i}}+\left(\omega_{i}^{-1 / 2} \cos \omega_{i} t\right) f_{j}(P, Q, t)\right]+O\left(\epsilon^{2}\right)
\end{aligned}
$$

where

$K(P, Q, t) \equiv H^{1}\left(\omega^{-1 / 2} Q \cos \omega t+\omega^{-1 / 2} P \sin \omega t, \quad-\omega^{1 / 2} Q \sin \omega t+\omega^{1 / 2} P \cos \omega t\right)$

and

$f_{i}(P, Q, t) \equiv F_{i}\left(\omega^{-1 / 2} Q \cos \omega t+\omega^{-1 / 2} P \sin \omega t, \quad-\omega^{1 / 2} Q \sin \omega t+\omega^{1 / 2} P \cos \omega t\right)$.

Now with the assumption that the frequencies are linearly independent over the integers, and that $H^{1}$ and $F_{i}$ are polynomials, and using the conditions (3.2) it can be shown after some calculations that if

$$
\widetilde{K}(u, v) \equiv \lim _{T \rightarrow \infty} T^{-1} \int_{0}^{T} K(u, v, t) d t
$$

then $\tilde{K}(u, v)$ takes the special form:

$$
\tilde{K}(u, v)=\tilde{K}\left[u_{1}, v_{1}, \frac{1}{2}\left(u_{1}^{2}+v_{1}^{2}\right), \frac{1}{2}\left(u_{2}^{2}+v_{2}^{2}\right), \cdots, \frac{1}{2}\left(u_{N}^{2}+v_{N}^{2}\right)\right]
$$

and quantities that arise from the dissipative forces have special forms:

$$
\begin{aligned}
& \lim _{r \rightarrow \infty} T^{-1} \int_{0}^{T}\left(\omega_{i}^{-1 / 2} \sin \omega_{i} t\right) f_{i}(u, v, t) d t=k_{j}(r) v_{i}-l_{j}(r) u_{i}, \\
& \lim _{T \rightarrow \infty} T^{-1} \int_{0}^{T}\left(\omega_{j}^{-1 / 2} \cos \omega_{i} t\right) f_{i}(u, v, t) d t=k_{j}(r) u_{i}+l_{j}(r) v_{i}, \quad j=1,2, \cdots N
\end{aligned}
$$

where $r_{k}=\frac{1}{2}\left(u_{k}^{2}+v_{k}^{2}\right)$ and where

$$
\begin{gathered}
k_{j}(r)=\operatorname{Re}\left[\left(2 r_{i}\right)^{-1 / 2} \lim _{T \rightarrow \infty} T^{-1} \int_{0}^{T} F_{j}\left(\omega^{-1}(2 r)^{1 / 2} \cos \omega \psi,-(2 r)^{1 / 2} \sin \omega \psi\right)\right. \\
\left.\cdot \exp \left(-i \omega_{i} \psi_{i}\right) d \psi_{i}\right] \\
l_{i}(r)=\operatorname{Im}\left[\left(2 r_{j}\right)^{-1 / 2} \lim _{T \rightarrow \infty} T^{-1} \int_{0}^{T} F_{i}\left(\omega^{-1}(2 r)^{1 / 2} \cos \omega \psi,-(2 r)^{1 / 2} \sin \omega \psi\right)\right. \\
\left.\cdot \exp \left(-i \omega_{i} \psi_{i}\right) d \psi_{i}\right] .
\end{gathered}
$$

The averaged equations corresponding to (3.9), (3.10), with the variables $Q$ and $P$ going to the variables $u$ and $v$ respectively under averaging, are as follows:

$$
\begin{aligned}
\dot{u}_{i} & =\epsilon\left[\tilde{K}_{, p_{i}}+k_{j}(r) v_{i}-l_{i}(r) u_{j}\right], \\
\dot{v}_{i} & =-\epsilon\left[\tilde{K}_{, u_{i}}+k_{i}(r) u_{i}+l_{j}(r) v_{i}\right] .
\end{aligned}
$$


To obtain (3.18) we have used (3.12), (3.14) and (3.15) and have taken advantage of the fact that the averaging operator and partial differentiation commute.

We now eliminate the constant term in the polynomial expression for $k_{j}(r)$ by letting

$$
\widetilde{\widetilde{K}}(u, v) \equiv \tilde{K}(u, v)+\frac{1}{2} \sum_{i=1}^{N} k_{j}(0)\left(u_{i}^{2}+v_{i}^{2}\right) .
$$

Since $\tilde{K}$ has the special form (3.13), (3.18) can be written thus:

$$
\begin{aligned}
& \dot{u}_{1}=\epsilon\left[\widetilde{\widetilde{K}}_{, v_{1}}+h_{1}(r) v_{1}-l_{1}(r) u_{1}\right], \\
& \dot{v}_{1}=-\epsilon\left[\widetilde{\widetilde{K}}_{, u_{1}}+h_{1}(r) u_{1}+l_{1}(r) v_{1}\right]
\end{aligned}
$$

and

$$
\begin{aligned}
& \dot{u}_{i}=\epsilon\left[\widetilde{\widetilde{K}}_{., j}+h_{i}(r) v_{j}-l_{j}(r) u_{i}\right] \\
& \dot{v}_{i}=-\epsilon\left[\tilde{\widetilde{K}}_{, u_{i}}+h_{j}(r) u_{i}+l_{i}(r) v_{j}\right]
\end{aligned}
$$

where $j=2,3, \cdots N$ and where $h_{j}(r)=k_{j}(r)-k_{i}(0), j=1,2, \cdots N$.

An inspection of the equations (3.19), (3.20) and (3.21) shows that they have critical points $u_{1}=u_{1}^{0}, v_{1}=v_{1}^{0}, u_{i}=v_{i}=0, j=2,3,4, \cdots N$ where $u_{1}^{0}, v_{1}^{0}$ are constants. It should be noted that, in general, the system (3.19), (3.20) and (3.21) will have several critical points, some stable and some unstable, but they all lie in the $\left(u_{1}, v_{1}\right)$ plane.

Let

$$
u_{1}=u_{1}^{0}+\xi, \quad v_{1}=v_{1}^{0}+\eta
$$

and let

$$
\begin{aligned}
M\left(\xi, \eta, r_{2}, \cdots r_{N}\right) & \equiv \widetilde{\widetilde{K}}\left(u_{1}, v_{1}, r_{2}, \cdots r_{N}\right) \\
& -M_{, \xi}\left(u_{1}^{0}, v_{1}^{0}, 0,0, \cdots, 0\right)-M_{, \eta}\left(u_{1}^{0}, v_{1}^{0}, 0,0, \cdots, 0\right)
\end{aligned}
$$

then (3.19) can be written as

$$
\xi=\epsilon\left[M_{, \eta}+h_{1}(r) \eta-l_{1}(r) \xi\right], \quad \dot{\eta}=-\epsilon\left[M_{. \xi}+h_{1}(r) \xi+l_{1}(r) \eta\right] .
$$

Now multiplying (3.20) by $u_{i}$ and (3.21) by $v_{i}$ and adding we have:

$$
\dot{r}_{i}=-\epsilon l_{i}(r) r_{i}, \quad j=2,3, \cdots N .
$$

We have thus been able to reduce the problem of finding a domain of attraction in the $2 N$ dimensional $(u, v)$ space to the problem of finding the domain of attraction in the $N+1$ dimensional $\left(u_{1}, v_{1}, r_{2}, r_{3}, \cdots r_{N}\right)$ space. Now (3.2) implies that $l_{i}(r) r_{i}>0$ for $r_{i} \neq 0$ and thus we see that the only critical points of the system (3.24), (3.25) are in the plane $r_{i}=0, j=2,3, \cdots N$. This fact, in turn, gives the desired result that the original averaged system (3.19), (3.20), (3.21) has critical points only in the $\left(u_{1}, v_{1}\right)$ plane.

It will now be assumed that the system (3.24) with $r_{2}=r_{3}=\cdots r_{N}=0$, that is the system:

$$
\begin{aligned}
& \dot{\xi}_{.}=\epsilon\left[M_{, \xi}^{0}+h_{1}^{0}\left(r_{1}\right) \eta-l_{1}^{0}\left(r_{1}\right) \xi\right], \\
& \dot{\eta}=-\epsilon\left[M_{, \eta}^{0}+h_{1}^{0}\left(r_{1}\right) \xi+l_{1}^{0}\left(r_{1}\right) \eta\right]
\end{aligned}
$$


where $M^{0}(\xi, \eta)=M(\xi, \eta, 0,0 \cdots 0), h_{1}^{0}\left(r_{1}\right)=h_{1}\left(r_{1}, 0,0, \cdots, 0\right)$ and $l_{1}^{0}\left(r_{1}\right)=l_{1}\left(r_{1}, 0,0, \cdots, 0\right)$ has its critical point $(0,0)$ asymptotically stable. If, furthermore, the linear variational equation for the origin of the system:

$$
\dot{\xi}=\epsilon M_{, \eta}^{0}, \quad \dot{\eta}=-\epsilon M_{, \xi}^{0}
$$

has distinct pure imaginary characteristic roots, then it can be shown that $M^{0}$ is a Liapunov function for the origin of (3.26).

Let a domain of attraction of the critical point $(0,0)$ of $(3.26)$ be the set

$$
S=\left\{(\xi, \eta): 0 \leq M^{0}(\xi, \eta)<C, C>0,(0,0) \varepsilon S\right\} .
$$

The constant $C$ is determined by the problem (3.26) and is different for each stable critical point in the $\left(u_{1}, v_{1}\right)$ plane. If $C<0$ we merely reverse the inequalities.

On $S, M^{0}$ is positive definite and

$$
\dot{M}^{0}=-\epsilon l_{1}^{0}\left(r_{1}\right)\left[M_{, \xi \xi}^{0}+M_{, \eta}^{0} \eta\right]+\epsilon h_{1}^{0}\left(r_{1}\right)\left[M_{, \xi \eta}^{0}-M_{,{ }^{\prime} \xi}^{0}\right]
$$

is negative definite on $S$.

Now let us try as a Liapunov function for system (3.24), (3.25) the function

$$
V\left(\xi, \eta, r_{2}, r_{3}, \cdots r_{N}\right)=M^{0}(\xi, \eta)+N\left(r_{2}, r_{3}, \cdots, r_{N}\right)
$$

where $N$ is a polynomial in $r_{2}, r_{3}, \cdots r_{2}$ the nature of which will be determined below.

Now if we compute the derivative of $V$ along the solutions of (3.24), (3.25) we have:

$$
\begin{aligned}
\dot{V}=\dot{M}_{0}+ & \epsilon-\left[l_{1}(r)-l_{1}^{0}\left(r_{1}\right)\right]\left[M_{, \xi \xi}^{0} \xi+M_{, \eta \eta}^{0} \eta\right] \\
& \left.+\left[h_{1}(r)-h_{1}^{0}\left(r_{1}\right)\right]\left[M_{. \xi}^{0} \eta-M_{. \eta}^{0} \xi\right]+\left[M_{, \xi}^{0} M_{, \eta}-M_{. \eta}^{0} M_{. \xi}\right]-\sum_{j=2}^{N} N_{{ }_{r_{i}}} l_{i}(r) r_{i}\right\} .
\end{aligned}
$$

Let

$$
\begin{aligned}
& P\left(r_{2}, r_{3}, \cdots r_{N}\right)=\sup _{\xi, \eta \varepsilon S}\left\{-\left[l_{1}(r)-l_{1}^{0}\left(r_{1}\right)\right]\left[M_{{ }^{\prime} \xi}^{0} \xi+M_{, \eta}^{0} \eta\right]\right. \\
& \left.+\left[h_{1}(r)-h_{1}^{0}\left(r_{1}\right)\right]\left[M_{, \xi}^{0} \eta-M_{. n}^{0} \xi\right]+\left[M_{{ }_{\xi}}^{0} M_{, \eta}-M_{{ }_{n}}^{0} M_{. \xi}\right]\right\}
\end{aligned}
$$

and thus $P(0,0, \cdots, 0)=0$.

Thus we have

$$
\dot{V} \leq \dot{M}^{0}+\epsilon\left[P\left(r_{2}, r_{3}, \cdots, r_{N}\right)-\sum_{i=2}^{N} N_{, r_{i}} l_{j}(r) r_{i}\right]
$$

for $\xi, \eta \varepsilon S$ and $r_{i} \geq 0, j=2,3, \cdots N$.

Now choose the coefficients of the polynomial $N\left(r_{2}, r_{3}, \cdots r_{N}\right)$ such that

$$
P\left(r_{2}, r_{3}, \cdots r_{N}\right)<\sum_{i} N_{, r_{i}} l_{i}(r) r_{i}
$$

for $r_{1}=\frac{1}{2}\left[\left(u_{1}^{0}+\xi\right)^{2}+\left(v_{1}^{0}+\eta\right)^{2}\right] \varepsilon S$ and $r_{i} \geq 0, j=2,3, \cdots N$, and such that $N$ is positive definite.

We thus have on the set

$$
S^{*}=\left\{\left(\xi, \eta, r_{2}, \cdots r_{N}\right): \xi, \eta \varepsilon S, \quad 0 \leq r_{i}<\infty, \quad j=2,3, \cdots N\right\},
$$

$V$ positive definite and $\dot{V}$ negative definite. 
Now consider the set

$\bar{\Omega}_{c-\delta}=\left\{\left(\xi, \eta, r_{2}, \cdots r_{N}\right): V\left(\xi, \eta, r_{2}, \cdots, r_{N}\right)<C-\delta\right.$,

origin $\varepsilon S, \bar{\Omega}_{c-\delta}$ simply connected\}.

In $\bar{\Omega}_{c-\delta}$ we have $V$ positive definite and $\dot{V}$ negative definite and the set $\bar{\Omega}_{c-\delta}$ satisfies the conditions of the Corollary to the main theorem. We thus have $\bar{\Omega}_{c-\delta}$ the domain of attraction of the almost periodic solution associated with the constant solution $\xi_{0}$.

In [10] the above procedure was employed for the study of a system with linear dissipation terms and cubic nonlinearities. In such systems the behavior in the principal plane is the same as that for Duffing's equation as given in [6]. One thus has, for the appropriate range of the excitation frequency, two stable and one unstable almost periodic solutions.

In [10] explicit values are given for the constant $C$ that occurs in equation (3.27) for the two stable almost periodic solutions.

Acknowledgment. The authors gratefully acknowledge valuable discussions with Dr. J. K. Hale during the early stages of this work.

\section{BIBLIOGRAPHY}

[1] J. K. Hale, Oscillations in nonlinear systems, McGraw-Hill, New York, 1963

[2] E. A. Coddington and N. Levinson, Theory of ordinary differential equations, McGraw-Hill, New York, 1955, Chaps. 13, 14

[3] N. M. Bogoliuboff and Yu. A. Mitropolskiy, Asymptotic methods in the theory of nonlinear oscillations, Gordon and Breach, New York, 1962

[4] Reference [3, Chap. 6]

[5] J. LaSalle and S. Lefschetz, Stability by Liapunov's second method, Academic Press, New York, 1961 , p. 59

[6] W. S. Loud and P. R. Sethna, Some explicit estimates for domains of attraction, J. Differential Eqs. (2) $2,158-172(1966)$

[7] Reference [3], Chap. 4

[8] P. R. Sethna, Coupling in certain classes of weakly nonlinear vibrating systems, in International Symposium on Nonlinear Differential Equations and Nonlinear Mechanics, J. P. LaSalle and S. Lefschetz, eds., Academic Press, New York, 1963

[9] Yu. A. Mitropolskiy, Problems of the asymptotic theory of nonstationary vibrations, Daniel Davey and Co., New York, 1965, Chaps. 4, 6

[10] P. R. Sethna, Domains of attraction of some quasi-periodic solutions, to be published in Proceedings of the International Symposium on Differential Equations and Dynamical Systems, Mayaguez, Puerto Rico December 1965 